\title{
A phase-I study of lapatinib in combination with foretinib, a c-MET, AXL and vascular endothelial growth factor receptor inhibitor, in human epidermal growth factor receptor 2 (HER-2)-positive metastatic breast cancer
}

Stephen K. Chia ${ }^{*}$, Susan L. Ellard ${ }^{2}$, Mihaela Mates ${ }^{3}$, Stephen Welch ${ }^{4}$, Catalin Mihalcioiu ${ }^{5}$, Wilson H. Miller Jr ${ }^{5}$,

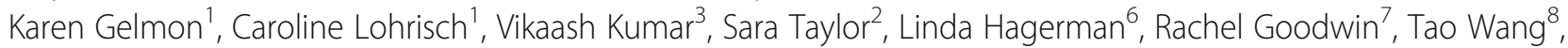
Shingo Sakashita ${ }^{8}$, Ming S. Tsao ${ }^{8}$, Elizabeth Eisenhauer ${ }^{3}$ and Penelope Bradbury ${ }^{8}$

\begin{abstract}
Background: The mechanisms of resistance to anti-human epidermal growth factor receptor 2 (HER 2) therapies are unclear but may include the tyrosine-protein kinase Met (c-Met), vascular endothelial growth factor (VEGF) and AXL pathways. Foretinib is an inhibitor of c-Met, VEGF receptor 2 (VEGFR-2), platelet-derived growth factor receptor beta (PDGFRB), AXL, Fms-like tyrosine kinase 3 (FLT3), angiopoiten receptor (TIE-2), RET and RON kinases. This phase Ib study sought to establish the associated toxicities, pharmacokinetics (PK) and recommended phase II doses (RP2D) of foretinib and lapatinib in a cohort of HER-2-positive patients with metastatic breast cancer (MBC).
\end{abstract}

Methods: Women with HER-2 positive MBC, Performance status (PS 0-2), and no limit on number of prior chemotherapies or lines of anti-HER-2 therapies were enrolled. A $3+3$ dose escalation design was utilized. Four dose levels were intended with starting doses of foretinib $30 \mathrm{mg}$ and lapatinib $750 \mathrm{mg}$ orally once a day (OD) on a 4-weekly cycle. Assessment of c-MET status from the primary archival tissue was performed.

Results: We enrolled 19 patients, all evaluable for toxicity assessment and for response evaluation. Median age was 60 years (34-86 years), 95\% were PS 0-1, 53\% were estrogen receptor-positive and 95\% had at least one prior anti-HER-2based regimen. The fourth dose level was reached (foretinib $45 \mathrm{mg} / \mathrm{lapatinib} 1250 \mathrm{mg}$ ) with dose-limiting toxicities of grade-3 diarrhea and fatigue. There was only one grade-4 non-hematological toxicity across all dose levels. There were no PK interactions between the agents. A median of two cycles was delivered across the dose levels (range 1-20) with associated progression-free survival of 3.2 months ( $95 \% \mathrm{Cl}$ 1.61-4.34 months). By immunohistochemical assessment with a specified cutoff, none of the 17 samples tested were classified as positive for c-Met.

Conclusions: The RP2D of the combined foretinib and lapatinib is $45 \mathrm{mg}$ and $1000 \mathrm{mg}$ PO OD, respectively. Limited activity was seen with this combination in a predominantly unselected cohort of HER-2-positive patients with MBC.

Keywords: HER-2, Lapatinib, c-Met inhibitor, Breast cancer, Pharmacokinetics

\footnotetext{
* Correspondence: schia@bccancer.bc.ca

'Medical Oncology, British Columbia Cancer Agency (BCCA), Vancouver, BC,

Canada

Full list of author information is available at the end of the article
} 


\section{Background}

The human epidermal growth factor receptor-2 (HER-2) proto-oncogene is both a prognostic factor and a predictive biomarker for anti-HER-2-targeted therapy. Prior to the advent of trastuzumab, a monoclonal antibody directed against the extra-cellular domain of the HER-2 receptor, the HER-2-positive subtype of breast cancer was associated with a worst prognosis compared to other subtypes of breast cancer [1,2]. Since the introduction of trastuzumab in the treatment of both early and advancedstage disease, the prognosis has been significantly altered with improved survival such that this subtype of breast cancer arguably now has one of the better prognoses when treated with anti-HER-2 therapies [3-5].

In the metastatic setting, despite improvements in outcome with HER-2 treatment, progression of disease and death still occur in the vast majority of patients $[6,7]$. Treatments designed to avoid or overcome resistance may provide improved outcomes for patients who develop resistance to therapy. Lapatinib is a small molecule tyrosine kinase inhibitor (TKI) of the HER family of receptors - in particular HER-1 and HER-2 [8]. Activity has been demonstrated for lapatinib in combination with hormonal therapy and with chemotherapy in HER2-positive metastatic breast cancer (MBC) $[9,10]$. Despite these demonstrated modest improvements in progression-free survival (PFS), there is either de novo resistance to lapatinib or it develops after therapy (acquired resistance). Little is known, however, about these potential mechanisms of resistance.

The tyrosine-protein kinase Met (c-Met) and the vascular endothelial growth factor (VEGF) pathways are proposed mechanisms of resistance to anti-HER-2-targeted therapies. C-Met is a transmembrane receptor tyrosine kinase to which its ligand, hepatocyte growth factor (HGF), binds. Potential oncogenic effects of activation of c-Met include proliferation, angiogenesis, migration and invasion - all hallmarks of a malignant process [11]. Pre-clinical studies have suggested activation of c-Met induces relative resistance to trastuzumab [12]. Likewise, activation of the VEGF pathway is an essential hallmark of cancer and has also been implicated as a resistance pathway to anti-HER-2-directed therapy [13]. Foretinib is an oral tyrosine kinase inhibitor of c-Met, VEGF receptor 2 (VEGFR-2), plateletderived growth factor beta (PDGFRB), AXL, Fms-like tyrosine kinase 3 (FLT3), angiopoiten receptor (TIE-2), RET and RON kinases, and therefore it is of interest to combine this with anti-HER-2 treatment to overcome putative resistance mechanisms.

The primary objectives of this study were to evaluate the safety profile, and establish the maximum administered dose of foretinib and lapatinib in a cohort of patients with HER-2-positive MBC. Secondary objectives included pharmacokinetics (PK) assessments of each agent, measurement of objective response rates, and evaluation of c-Met in the primary archival tumor specimen.

\section{Methods}

Female patients with HER-2-positive breast cancer (immunohistochemical grade 3+ or fluorescence in situ hybridization ratio $\geq 2.0$ as per local assessment), as per American Society of Clinical Oncology/College of American Pathologists (ASCO/CAP) guidelines during the time course of the study, who had incurable loco-regional recurrent or metastatic disease, were enrolled across five Canadian cancer centers. There was no limit on the number of prior systemic therapies (hormonal or chemotherapy) or number of prior anti-HER-2-targeted therapies (including lapatinib) delivered prior to study entry.

Patients were to have a baseline performance status of $0-2$, and adequate hematological, hepatic and renal function. Specifically, the eligibility criteria were creatinine $\leq 1.2$ times the upper limit of normal (ULN), total bilirubin $\leq 1.2$ times the ULN and aspartate aminotransferase (AST)/alanine aminotransferase (ALT) $\leq 2$ times the ULN. A baseline left ventricular ejection fraction of $\geq 50 \%$ was also required for study entry. Exclusion criteria included prior exposure to a c-Met inhibitor or to a VEGFR inhibitor; previous history of thromboembolic disease within 6 months prior to study entry; uncontrolled hypertension, active infection, untreated brain metastasis or leptomeningeal disease or serious cardiovascular disease. Measurable disease was not a requirement for the dose escalation phase of the study. Last, tumor specimens were required from all enrolled patients, comprising at minimum a formalin-fixed paraffinembedded (FFPE) sample from the primary tumor.

This was an open-label multi-center phase I dose escalation study of the combination of foretinib and lapatinib. The study utilized a standard $3+3$ design with four dose levels. The starting dose of each agent (foretinib 30 $\mathrm{mg} /$ lapatinib $750 \mathrm{mg}$ ) in dose-level 1 was $50 \%$ of each agent given as monotherapy because of the potential for overlapping toxicities. Each agent was given orally (PO) once a day (OD), with 4 weeks duration of therapy defined as one cycle. For the first cycle of each dose level, lapatinib was started on day 1 and foretinib started on day 3. Thereafter they were taken continuously together.

No new patients were entered at an escalated dose level until at least three patients had completed one full treatment cycle at the previous level and the maximum administered dose had not been reached. As an added precaution, the first patient treated at dose-level 1 (DL 1) must have completed one cycle prior to the second and third patient commencing therapy on that first dose level. If no dose-limiting toxicity (DLT) was observed in the first three patients during the first cycle of therapy of each dose 
level, then the next higher dose level opened to recruitment (Table 1). If one (but not two or more) of the first three patients at a dose level experienced DLT, then an additional three patients were added at that dose level. The maximum administered dose (MAD) was defined as the dose level at which $\geq 2 / 6$ or $\geq 2 / 3$ patients experienced dose-limiting toxicity. The next lower dose below the MAD was then declared the recommended phase II dose (RP2D).

Adverse events were assessed using Common Terminology Criteria for Adverse Events (CTCAE) version 4.0. DLT was defined as any of the following occurring during cycle 1 of each dose level: (a) grade- 3 or worse non-hematologic toxicity (excluding grade- 2 alopecia or inadequately controlled/managed diarrhea, nausea/vomiting, rash or hypertension) or hematologic toxicities of grade-4 neutropenia for $\geq 7$ days, grade-3/4 neutropenia with fever, grade- 4 thrombocytopenia or anemia or any grade of thrombocytopenia associated with bleeding; (b) the inability to administer cycle-2 treatment within 14 days of the planned dose (i.e. cycle-2 treatment delayed by more than 2 weeks) due to persisting grade-2 drug-related toxicity.

Patients were assessed clinically on day 1 of each cycle with standard hematological and biochemical blood work performed weekly during cycle 1 and then on day 1 of each subsequent cycle. Radiological imaging of the tumor burden was performed at baseline and at the end of every second cycle. Objective response was assessed using Response Evaluation Criteria In Solid Tumors (RECIST) 1.1. Assessment of the left ventricular ejection function (LVEF) was performed following every third cycle of therapy. Limited PK sampling was performed on day 1 and day 29 of cycle 1 only. Patients received treatment until evidence of progressive disease, unacceptable toxicity or elective withdrawal from study.

\section{C-Met assessment}

Archival FFPE material from all consenting study participants was requested for immunohistochemical analysis

Table 1 Dose levels of lapatinib and foretinib

\begin{tabular}{llll}
\hline Dose level & $\begin{array}{l}\text { Foretinib/lapatinib } \\
\text { dose }\end{array}$ & $\begin{array}{l}\text { Number of } \\
\text { cycles }\end{array}$ & $\begin{array}{l}\text { Number of } \\
\text { patients }\end{array}$ \\
\hline 1 & $30 \mathrm{mg} / 750 \mathrm{mg}$ & 2 & 2 \\
& & 20 & 1 \\
2 & 2 & 2 \\
3 & $30 \mathrm{mg} / 1000 \mathrm{mg}$ & 5 & 1 \\
& & 2 & 2 \\
4 & $45 \mathrm{mg} / 1000 \mathrm{mg}$ & $3-4$ & 4 \\
& & $1-2$ & 4 \\
& $45 \mathrm{mg} / 1250 \mathrm{mg}$ & 4 & 1 \\
& & 6 & 2 \\
\hline
\end{tabular}

(IHC) of c-Met using monoclonal antibodies against c-Met (clone SP44; Ventana, Tucson, AZ, USA). The staining was conducted using the Ventana BenchMark Autostainer as per the company's recommended protocol (SP44) as reported previously [14]. Expression levels of c-Met were assessed semi-quantitatively using the standard $H$ score $\geq 200$.

\section{Statistics}

This was a phase I study with the primary objective designed to determine the recommended phase II dose of foretinib in combination with lapatinib in patients with recurrent or metastatic HER-2+ breast cancer. The final sample size was dependent upon the number of dose levels required to reach the recommended phase II dose. Once the recommended phase II dose was established, there was an option to enroll up to 15 additional patients to further evaluate toxicity, response and correlative studies of the combination, should an efficacy signal emerge (operationally defined as the observation of at least one objective response) from the dose escalation component of the study.

\section{Role of the funding source}

The study was approved by the local/provincial Research Ethics Board (REB) governing each participating center. All participating subjects signed an informed consent document.

\section{Results}

A total of 19 patients were enrolled across five Canadian cancer centers. The baseline clinical and pathological details of these 19 patients are outlined in Table 2. The majority of patients had performance status 0-1 (95\%), had received prior anti-HER-2 therapy for MBC (95\%), had received prior chemotherapy (89\% including 10/19 who had had three or more prior chemotherapy regimens), and had visceral disease involvement (84\%). Of the 19 patients, 8 had been treated both with trastuzumab and lapatinib prior to enrollment into the study.

No DLT was observed during cycle 1 across the first three dose levels (DL 1-3). The third enrolled patient at DL 4 experienced persistent grade- 3 fatigue and persistent grade-2 nausea, vomiting, diarrhea and myalgia requiring dose interruption and dose reduction, resulting in a DLT. As a consequence, an additional four subjects were enrolled at DL 4 (foretinib $45 \mathrm{mg}$ /lapatinib 1250 $\mathrm{mg})$. At this expanded dose level, two of these four patients also experienced a DLT in cycle 1 . One patient developed grade-3 fatigue and another developed grade3 diarrhea - both requiring dose reductions. As a consequence, the maximum administered dose was declared at DL 4. Per protocol, a further three patients were enrolled at DL 3. No DLTs were observed in these three 
Table 2 Baseline characteristics of the study population $(n=19)$

\begin{tabular}{ll}
\hline Characteristic & Value \\
\hline Age, years, median (range) & $60(34-86)$ \\
Performance status, $n$ & \\
0 & 9 \\
1 & 9 \\
2 & 1 \\
Measurable disease, $n$ (\%) & $16(84)$ \\
Prior therapy, $n$ & \\
Chemotherapy & 17 \\
Hormonal therapy & 7 \\
HER-2 inhibitor & 18 \\
Radiotherapy & 15 \\
ER-positive, $n$ (\%) & $10(53 \%)$ \\
HER-2 positive, $n$ (\%) & $19(100 \%)$ \\
Number of sites of disease, $n$ (\%) & \\
1 & $5(26 \%)$ \\
2 & $2(11 \%)$ \\
3 & $6(32 \%)$ \\
$\geq 4$ & $6(32 \%)$ \\
Site of disease, $n$ (\%) & $10(53 \%)$ \\
Bone & $2(11 \%)$ \\
Brain tissue & $16(84 \%)$ \\
\hline$n$ number of patients, HER-2 human epidermal growth factor receptor $2, E R$ \\
estrogen receptor & \\
\hline & \\
\hline &
\end{tabular}

additional enrolled patients at this dose level. As such, the RP2D of the combination was deemed to be foretinib 45 mg PO OD and lapatinib 1000 mg PO OD (DL 3).

The most common grade 1-4 adverse events with a frequency $\geq 20 \%$ across DL $3-4$ are outlined in Table 3. Most notably, diarrhea, fatigue, elevated liver enzymes, nausea and proteinuria were observed. There was an occurrence of a grade- 3 thromboembolic event in two patients and one patient developed both grade- 3 proteinuria and grade- 3 peripheral edema that was determined to be treatment-related. There was complete resolution of the proteinuria and peripheral edema with discontinuation of foretinib. The spectrum of toxicities was in keeping with target inhibition of c-Met, VEGF, EGFR and HER-2.

Limited sampling for PK was undertaken in this study. The PK data of the drugs in combination was similar to historical data on the use of the drugs as single agents, suggesting no significant apparent interactions (Additional file 1: Table S1A and Table S1B).

Among the 19 enrolled patients, there were no documented responses according to the RECIST criteria. In 6
Table 3 Frequent $(\geq 20 \%)$ reported adverse events across dose levels 3-4 that were considered related to both foretinib and lapatinib

\begin{tabular}{|c|c|c|c|}
\hline & $\begin{array}{l}\text { Adverse events } \\
\text { Grade 1-2 }\end{array}$ & Grade 3 & Grade 4 \\
\hline \multicolumn{4}{|l|}{ Dose level $3(n=6)$} \\
\hline Diarrhea & 6 & & \\
\hline Nausea & 6 & & \\
\hline Vomiting & 4 & 1 & \\
\hline Fatigue & 5 & & \\
\hline Anorexia & 5 & & \\
\hline Proteinuria & 4 & & \\
\hline Thromboembolic & 1 & 2 & \\
\hline Anemia & 4 & & \\
\hline AST and/or ALT elevation & $5 / 6$ & & \\
\hline \multicolumn{4}{|l|}{ Dose level $4(n=7)$} \\
\hline Diarrhea & 2 & 5 & \\
\hline Nausea & 7 & & \\
\hline Vomiting & 5 & & \\
\hline Fatigue & 5 & 2 & \\
\hline Anorexia & 5 & & \\
\hline Proteinuria & 2 & 1 & \\
\hline Edema (limbs) & 2 & & \\
\hline Myalgia & 3 & & \\
\hline Hypertension & 4 & & \\
\hline Anemia & 2 & & \\
\hline AST and/or ALT elevation & $5 / 4$ & $2 / 3$ & \\
\hline
\end{tabular}

AST aspartate aminotransferase, ALT alanine aminotransferase

patients (32\%) the best response was stable disease (median duration 4.3 months, range 3.0-10.9 months) and in 12 patients it was progressive disease; response could not be evaluated in 1 patient. The median PFS in the study cohort was 3.2 months (95\% CI 1.61-4.34 months) (Fig. 1). Among the 19 patients enrolled, c-Met was evaluated by IHC in 14 patients (there were no results for 5 patients, 4 patients had no target lesions and response was not evaluable in 1 patient). With the predefined cut point $\geq 200$ (considered positive for c-Met), none of the patients had tumors positive for c-Met, 15 patients had tumors that were negative for c-Met and in 4 patients the status was unknown or not assessable (due to insufficient tumor tissue from 2 patients, and to status not being assessed in the other 2 patients because the tissue had been returned to the original pathology laboratory). The best tumor shrinkage and c-Met status are summarized in a waterfall plot (Fig. 2).

\section{Discussion}

Despite the initial dramatic benefits seen with adjuvant trastuzumab concurrent or sequential to chemotherapy, 
Progression-Free Survival

(events include all deaths unless censored for other trt)

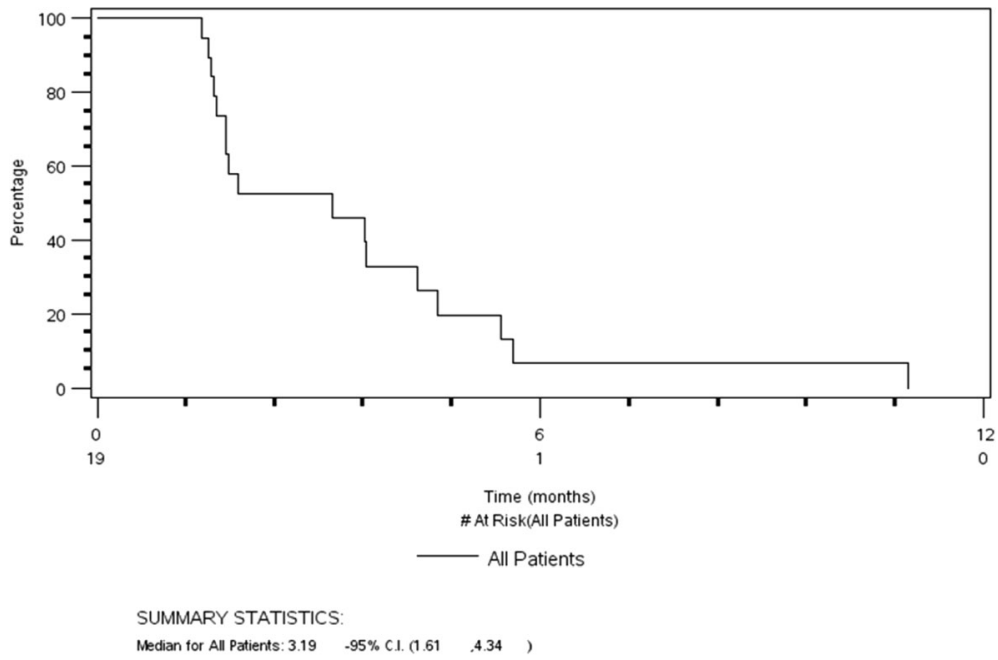

Fig. 1 Progression-free survival (PFS). trt treatment

relapses are unfortunately still occurring over time. At the first analysis of the pivotal NSABP B-31 and NCCTG N9831, with 2 years of median follow up, the disease free survival (DFS) was 87.1\% [14]. Now, with longer follow up, the rate of DFS at year 10 was $73.7 \%$ [15].

In advanced-stage disease, despite significant improvements in PFS and overall survival with dual HER-2 antibody blockade (pertuzumab and trastuzumab) and with an antibody drug conjugate (TDM1), progression of disease and death ultimately occurs in the vast majority of patients $[6,7,16]$. The CLEOPATRA trial demonstrated the combination of pertuzumab, trastuzumab and docetaxel was superior to trastuzumab and docetaxel in both
PFS (median PFS 18.7 months vs 12.4 months) and overall survival (OS) (median OS 56.5 months vs 40.8 months), respectively $[6,16]$. The EMELIA trial demonstrated that the antibody drug conjugate (TDM1) was superior to the combination of lapatinib and capecitabine in both PFS (median PFS 9.6 months vs 6.4 months) and OS (median OS 30.9 months vs 25.1 months) respectively [7]. Though these two landmark trials have established the preferred treatment algorithm in first-line and second-line disease, additional therapeutic agents are still needed in the management of HER-2-positive MBC, particularly those that may circumvent or overcome resistance to targeted HER-2 therapy.

1198 - by Cmet (Cutpoint 200)

( $n=14$ evaluable patients)

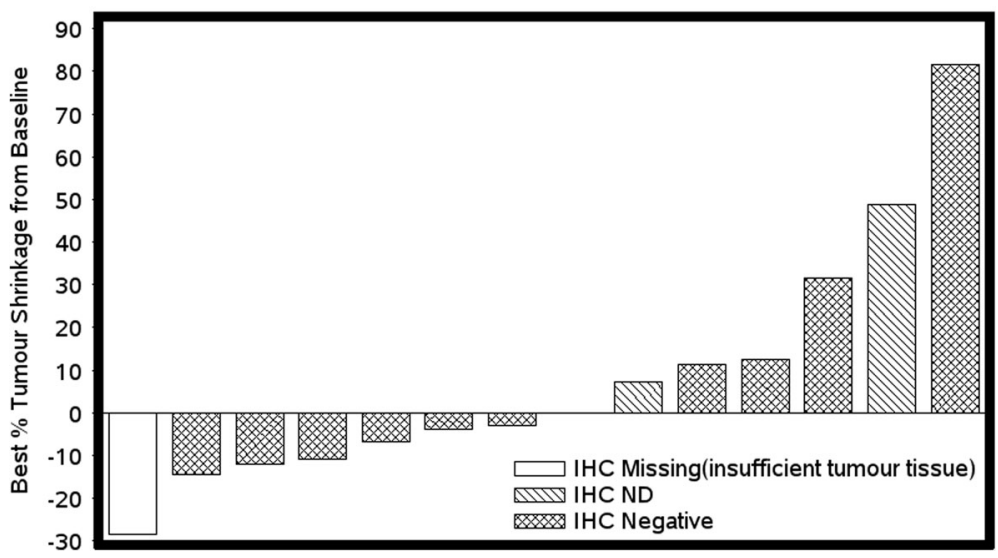

Fig. 2 Best tumor shrinkage based on tyrosine-protein kinase Met expression. IHC immunohistochemial analysis 
The combination of TDM1 and pertuzumab was studied in the phase III MARIANNE trial comparing it to TDM1 alone or docetaxel and trastuzumab as firstline therapy for HER-2-positive $\mathrm{MBC}$, unfortunately with no improvements in PFS demonstrated for the TDM1 and pertuzumab combination arm relative to the other arms investigated [17]. As mutations in the phosphatidylinositol 3 kinase (PIK3CA) exons 9 and 20 gene and/or loss of the tumor suppressor gene PTEN occurs frequently in breast cancer with over-expression of HER-2 and is associated with a poorer outcome and relative trastuzumab resistance, investigations into blocking this pathway in combination with trastuzumab have been investigated. Everolimus is a mammalian target of rapamycin (mTOR) inhibitor with demonstrated clinical activity in combination with hormonal therapy in ER+ MBC [18]. The BOLERO-1 investigated the combination of everolimus with paclitaxel and trastuzumab vs placebo with paclitaxel and trastuzumab as first-line therapy in HER-2-positive MBC. The BOLERO-3 investigated the combination of everolimus with vinorelbine and trastuzumab vs placebo with vinorelbine and trastuzumab as second-line therapy or later in HER-2-positive MBC. In a combined translational study there was correlation between greater benefit and everolimus in the cohort with PIK3CA mutation, with a hazard ratio of 0.67 (95\% CI 1.45-1.0) [19]. Though supporting the hypothesis for current clinical trials of PIK3CA inhibitors in combination with anti-HER-2 therapy, the clinical utility of evolimus and trastuzumab with chemotherapy is limited by their toxicities and the changing landscape of HER-2 agents.

Lapatinib is a small molecule tyrosine kinase inhibitor of HER-1 and HER-2. The proposed mechanism of antitumor activity is the inhibition of the downstream pathways of PI3K/Akt and mitogen-activated protein kinase (MAPK), induction of apoptosis and inhibition of proliferation, which results from inhibiting phosphorylation of these receptors by lapatinib [20]. Lapatinib in combination with capecitabine in metastatic HER-2-positive breast cancer following prior trastuzumab exposure has demonstrated improved PFS to 8.4 months for the combination vs 4.4 months for capecitabine alone [10]. However, no overall survival difference between the regimens was seen, likely in part due to cross-over after the study was halted. Inconsistent results have been demonstrated for the addition of lapatinib to hormonal therapy in hormone-receptor-positive HER-2-positive $\mathrm{MBC}$, with some improvement in PFS in combination with letrozole, but no benefit seen in combination with fulvestrant $[9,21]$. Last, the combination of lapatinib and trastuzumab demonstrated superior efficacy compared to lapatinib alone in a trial of HER-2-positive $\mathrm{MBC}$ that was refractory to trastuzumab [22].
Mechanisms of resistance to anti-HER-2 therapies, in particular the small molecule TKIs are widely speculated but not validated in clinical practice. Broadly, the main mechanisms of resistance to lapatinib can be categorized into activation of compensatory pathways, mutations of the HER-2 tyrosine kinase (TK) domain, and gene amplification. Within the category of activation of compensatory pathways, the c-Met and AXL receptor tyrosine kinases (RTKs) have been implicated as potential key mediators. The c-Met TK is a transmembrane receptor for hepatocyte growth factor (HGF), which upon activation is associated with increased proliferation, cell invasion and evasion of apoptosis [23, 24]. Expression of c-Met has been associated with resistance to both trastuzumab and lapatinib [25]. More importantly, in a pre-clinical study of the combination of a c-Met TKI and lapatinib in an HER-2positive gastric cell line, resistance to lapatinib was overcome with restoration of growth inhibition [26].

AXL is a transmembrane receptor with an intracellular kinase domain that has structural similarities to c-Met. Mechanistically, activation of the pathway engages the PI3K regulatory subunit p85 to activate the PI3K pathway, and by doing so bypasses upstream blockade. Activation of the pathway has been associated with clinical resistance to TKIs such as imatinib for gastrointestinal stromal tumors (GIST), epidermal growth factor receptor (EGFR) TKIs in non-small cell lung cancer and to lapatinib in pre-clinical breast cancer models [26, 27]. A multi-kinase AXL inhibitor has demonstrated restoration of sensitivity to both lapatinib and trastuzumab in a breast cancer model with acquired resistance to lapatinib [28].

A phase II study of single-agent foretinib in advancedstage triple-negative breast cancer (TNBC) has been performed and recently published by our group [29]. A daily dose of foretinib of $60 \mathrm{mg}$ PO was delivered in 45 patients (37 evaluable) with the most common grade- 3 or greater toxicities seen as hypertension (49\%), diarrhea (7\%), nausea (4\%) and fatigue (4\%). Though the response rate was low $(4.7 \%)$, a stable disease rate of $33 \%$ was demonstrated (median duration 5.4 months). There was no correlation between Met status of the tissue or in circulating tumor cells (CTC) and clinical activity.

To our knowledge, this is the first published clinical study of a combination of an anti-HER-2 agent with a c-Met inhibitor in breast cancer. Overall the toxicity profile was in keeping with known toxicities of both agents, with the most common grade 3-4 adverse events occurring at the maximal administered dose (DL 4), and consisting of fatigue and diarrhea. Other prominent grade-2 toxicities at this higher dose level were anorexia, nausea, vomiting and musculoskeletal symptoms. Two subjects developed thromboembolic disease, and c-Met inhibitors are known to increase the rate of thrombosis $[30,31]$. In the entire study population, however, we did 
not demonstrate any responses and the median PFS of 3.2 months was disappointing. Though we had intended an exploratory analysis of response or clinical benefit in c-Met-positive tumors, unfortunately none of the samples that were collected within the study population were classified as c-Met-positive by IHC and by the defined staining cutoff.

Beyond the limited efficacy in this study, other limitations of our study included a relatively small sample size at the RP2D $(n=6)$, lack of assessment of c-Met or other biomarkers on a more contemporary tumor specimen reflective of the metastatic phenotype, lack of pharmacodynamic modulation assessment for the combination of drugs, and the broad inclusion of any HER-2-positive disease. The absence of demonstration of c-Met expression in our population also may cast doubt on the hypothesis of this being an important target for resistance modulation to anti-HER-2 therapy.

\section{Conclusions}

In our phase-I study, we have determined the recommended doses for consideration in future studies of the combination of foretinib and lapatinib to be $45 \mathrm{mg}$ and $1000 \mathrm{mg}$ PO daily, respectively. At our recommended phase-II dose level, the combination appears relatively tolerable apart from modest risks of diarrhea, fatigue, anorexia and nausea. There was limited efficacy (median PFS 3.2 months (95\% CI 1.61-4.34 months)) in our study population. Future studies of this combination, if considered, should likely only include a population that demonstrates activity of either the c-Met and/or AXL pathways as drivers of either primary or acquired resistance to anti-HER-2-directed therapies.

\section{Additional file}

Additional file 1: Table S1A. Pharmacokinetics of lapatinib. Table S1B. Pharmacokinetics of foretinib. (DOCX $558 \mathrm{~kb}$ )

\section{Abbreviations}

ALT: Alanine aminotransferase; AST: Aspartate aminotransferase; CCTG: Canadian Clinical Trials Group; c-Met: Tyrosine-protein kinase Met; DL: Dose level; DLT: Dose-limiting toxicity; EGFR: Epidermal growth factor receptor; FFPE: Formalin-fixed paraffin-embedded; FLT3: Fms-like tyrosine kinase 3; HER-2: Human epidermal growth factor 2; HGF: Hepatocyte growth factor; MAD: Maximal administered dose; MBC: Metastatic breast cancer; mTOR: Mammalian target of rapamycin; OS: Overall survival; PDGRFB: Platelet-derived growth factor receptor beta; PFS: Progression-free survival; PI3K: Phosphatidylinositol 3 kinase; PK: Pharmacokinetics; PO: Per oral; PTEN: Phosphatase and tensin homolog; RP2D: Recommended phase II dose; TK: tyrosine kinase; TKI: Tyrosine kinase inhibitor; VEGF: Vascular endothelial growth factor

\section{Acknowledgements}

The Canadian Cancer Trials Group (CCTG) is supported by the Canadian Cancer Society Research Institute to the Canadian Cancer Trials Group (Grant \#021039). PAB is funded by a Cancer Care Ontario Research Chair in Experimental Therapeutics. Fellows (PAB and RG) are funded by AstraZeneca - CCTG Drug Development Fellowship and the Terry Fox Foundation
Training Program in Transdisciplinary Cancer Research in partnership with ClHR, Canadian Cancer Trials Group Development Fellowship.

\section{Funding}

Glaxo-Smith Kline provided the study drugs (both lapatinib and foretinib) free of charge to the investigators and patients for the purposes of this study and also provided financial support for the study conduct.

\section{Availability of data and materials}

Supplemental tables and supporting data are available upon request from CCTG.

\section{Authors' contributions}

The CCTG was responsible for trial development, conduct, creation of the study database and analysis and had the final responsibility for the manuscript. Study concept development: SC, PB, RG and EE. Accrual and conduct of the study: the CCTG and all authors. Analysis of data: CCTG, SC, $\mathrm{LH}, \mathrm{EE}, \mathrm{MT}, \mathrm{SS}, \mathrm{TW}$ and PB. Manuscript writing and review of manuscript: all authors. All authors read and approved the final manuscript.

\section{Competing interests}

SLE declares owning stock in Glaxo-Smith Kline. All other investigators have declared no conflict of interests.

Consent for publication

All authors and the CCTG consent for publication.

\section{Ethical approval and consent to participate}

The study was approved by the local/provincial Research Ethics Board (REB) governing each participating center. All participating subjects signed the informed consent document.

\section{Publisher's Note}

Springer Nature remains neutral with regard to jurisdictional claims in published maps and institutional affiliations.

\section{Author details}

${ }^{1}$ Medical Oncology, British Columbia Cancer Agency (BCCA), Vancouver, BC, Canada. ${ }^{2}$ Medical Oncology, BCCA, Kelowna, BC, Canada. ${ }^{3}$ Queen's University and Cancer Centre of South Eastern Ontario at Kingston General Hospital, Kingston, ON, Canada. ${ }^{4}$ London Regional Cancer Program, London, ON, Canada. ${ }^{5}$ Jewish General Hospital and Rossy Cancer Network, McGill University, Montreal, QC, Canada. ${ }^{6}$ Canadian Cancer Trials Group, Kingston, ON, Canada. ${ }^{7} T$ The Ottawa Hospital Cancer Centre, Ottawa, ON, Canada. ${ }^{8}$ Princess Margaret Cancer Centre and University Health Network, University of Toronto, Toronto, ON, Canada.

Received: 11 July 2016 Accepted: 16 March 2017

Published online: 02 May 2017

\section{References}

1. Slamon DJ, Clark GM, Wong SG, et al. Human breast cancer: correlation of relapse and survival with amplification of the HER-2/neu oncogene. Science. 1987;235:177-82.

2. Sorlie T, Perou CM, Tibshirani R, et al. Gene expression patterns of breast carcinomas distinguish tumor subclasses with clinical implications. Proc Natl Acad Sci USA. 2001;98:10869-74.

3. Gianni L, Dafni U, Gelber RD, et al. Treatment with trastuzumab for 1 year after adjuvant chemotherapy in patients with HER2-positive early breast cancer: a 4-year follow-up of a randomised controlled trial. Lancet Oncol. 2011;12:236-44.

4. Perez EA, Romond EH, Suman VJ, et al. Four-year follow-up of trastuzumab plus adjuvant chemotherapy for operable human epidermal growth factor receptor 2-positive breast cancer: joint analysis of data from NCCTG N9831 and NSABP B-31. J Clin Oncol. 2011;29:3366-73.

5. Seal MD, Speers C, O'Reilly $S$, et al. Outcomes of women with early stage breast cancer receiving adjuvant trastuzumab. Curr Oncol. 2012;19:197-201.

6. Baselga J, Cortes J, Kim SB, et al. Pertuzumab plus trastuzumab plus docetaxel for metastatic breast cancer. N Engl J Med. 2012;366:109-19.

7. Verma S, Miles D, Gianni L, et al. Trastuzumab emtansine for HER2-positive advanced breast cancer. N Engl J Med. 2012;367:1783-91. 
8. Konecny GE, Pegram MD, Venkatesan N, et al. Activity of the dual kinase inhibitor lapatinib (GW572016) against HER-2 over-expressing and trastuzumab treated breast cancer cells. Cancer Res. 2006;64:1630-41.

9. Johnston S, Pippen J, Pivot $X$, et al. Lapatinib combined with letrozole versus letrozole and placebo as first-line therapy for postmenopausal hormone receptor-positive metastatic breast cancer. J Clin Oncol. 2009;27:5538-46.

10. Geyer CE, Forster J, Lindquist D, et al. Lapatinib plus capecitabine for HER2positive advanced breast cancer. N Engl J Med. 2006;355:2733-43.

11. Hanahan D, Weinberg RA. Hallmarks of cancer: the next generation. Cell. 2011;144:646-74.

12. Shattuck DL, Miller JK, Carraway $\mathrm{KL}$, et al. Met receptor contributes to trastuzumab resistance of Her2-overexpressing breast cancer cells. Cancer Res. 2008;68:1471-7.

13. Konecny GE, Meng YG, Untch M, et al. Association between HER-2/neu and vascular endothelial growth factor expression predicts clinical outcome in primary breast cancer patients. Clin Cancer Res. 2004;10:1706-16.

14. Chau NG, Perez-Ordonex B, Zhang K, et al. The association between EGFR variant III, HPV, p16, c-MET, EGFR gene copy number and response to EGFR inhibitors in patients with recurrent or metastatic squamous cell carcinoma of the head and neck. Head Neck Oncol. 2011;3:11.

15. Perez EA, Romond EH, Suman VJ, et al. Trastuzumab plus adjuvant chemotherapy for human epidermal growth factor receptor 2 positive breast cancer: planned joint analysis of overall survival from NSABP B-31 and NCCTG N9831. J Clin Oncol. 2014;32:3744-52.

16. Swain SM, Cortes J, Kim SB, et al. Pertuzumab, trastuzumab, and docetaxel for HER2 positive metastatic breast cancer (CLEOPATRA study): overall survival results from a randomised, double-blind, placebo-controlled, phase 3 study. Lancet Oncol. 2013;14:461-71.

17. Ellis PE, Barrios CH, Eiermann W, et al. Phase III, randomized study of trastuzumab emtansine \pm pertuzumab vs trastuzumab + taxane for first-line treatment of HER2-positive MBC: primary results from the MARIANNE study. J Clin Oncol. 2015;33(suppl; abstr 507).

18. Baselga J, Campone M, Piccart M, et al. Everolimus in post menopausal hormone receptor positive advanced breast cancer. N Engl J Med. 2012;366:520-9.

19. Slamon D, Hurvitz S, Chen D, et al. Predictive biomarkers of everolimus efficacy in HER2+ advanced breast cancer. Combined exploratory analysis from BOLERO-1 and BOLERO-3. J Clin Oncol. 2015;33(suppl; abstr 512).

20. Medina PJ, Goodin S. Lapatinib: a dual inhibitor of human epidermal growth factor receptor tyrosine kinases. Clin Ther. 2008;30:1426-47.

21. Burstein HJ, Cirrincione CT, Barry ST, et al. Endocrine therapy with or without inhibition of epidermal growth factor receptor and human epidermal growth factor receptor 2: a randomized, double-blind, placebocontrolled phase III trial of fulvestrant with or without lapatinib for postmenopausal women with hormone receptor-positive advanced breast cancer-CALGB 40302 (Alliance). J Clin Oncol. 2014;32:3959-66.

22. Blackwell KL, Burstein HJ, Storniolo AM, et al. Randomized study of lapatinib alone or in combination with trastuzumab in women with ErbB2-positive, trastuzumab-refractory metastatic breast cancer. J Clin Oncol. 2010;28:1124-30.

23. Comoglio PM, Giordano G, Trusolino L, et al. Drug development of MET inhibitors: targeting oncogene addiction and expedience. Nat Rev Drug Discov. 2008;7:504-16.

24. Pohlmann PR, Mayer IA, Mernaugh $\mathrm{R}$, et al. Resistance to trastuzumab in breast cancer. Clinical Cancer Res. 2009;15:7479-91.

25. Chen CT, Kim H, Liska D, et al. MET activation mediates resistance to lapatinib inhibition of HER2-amplified gastric cancer cells. Mol Cancer Ther 2012;11:660-9.

26. Mahadevan D, Cooke L, Riley C, et al. A novel tyrosine kinase switch is a mechanism of imatinib resistance in gastrointestinal stromal tumors. Oncogene. 2007;26:3909-19.

27. Zhang $Z$, Lee JC, Lin L, et al. Activation of the AXL kinase causes resistance to EGFR-targeted therapy in lung cancer. Nat Genet. 2012;44:852-60.

28. Liu L, Shi H, Liu Y, et al. Synergistic effects of foretinib with HER-targeted agents in MET and HER1- or HER2-coactivated tumor cells. Mol Cancer Ther. 2011;10:518-30.

29. Rayson D, Lupichuk S, Potvin K, et al. Canadian Cancer Trials Group IND197: a phase II study of foretinib in patients with estrogen receptor, progestrone receptor and HER-2 negative recurrent or metastatic breast cancer. Breast Cancer Res Treat. 2016;157:109-16.
30. Wagner AJ, Goldberg JM, Dubois SG, et al. Tivantinib (ARQ 197), a selective inhibitor of MET, in patients with microphthalmia transcription factor associated tumors: results of a multicentre phase 2 trial. Cancer. 2012;118:5894-902.

31. Romond EH, Perez EA, Bryant J, et al. Trastuzumab plus adjuvant chemotherapy for operable HER2-positive breast cancer. N Engl J Med. 2005;353:1673-84

\section{Submit your next manuscript to BioMed Central and we will help you at every step:}

- We accept pre-submission inquiries

- Our selector tool helps you to find the most relevant journal

- We provide round the clock customer support

- Convenient online submission

- Thorough peer review

- Inclusion in PubMed and all major indexing services

- Maximum visibility for your research

Submit your manuscript at www.biomedcentral.com/submit
C Biomed Central 